\title{
Visitação aberta em unidade de terapia intensiva neonatal: percepções da equipe de enfermagem
}

\author{
Open visitation in a neonatal intensive care unit: nursing team's perceptions \\ Visitación abierta en unidad de terapia intensiva neonatal: percepciones del equipo de enfermería
}

\author{
Fábio Luiz Banhara'; Francely Tineli Farinhall; Tatiane Henrique III; Ana Paula Ribeiro Razeralv; \\ Nadja Guazzi Arenales Alves ${ }^{v}$; Armando dos Santos Trettene ${ }^{v l}$
}

\begin{abstract}
RESUMO
Objetivo: compreender a experiência de profissionais de enfermagem sobre a visitação aberta em uma unidade de terapia intensiva neonatal (UTIN), antes e após sua implementação. Método: estudo descritivo, qualitativo, desenvolvido em uma maternidade pública situada em Bauru, São Paulo, Brasil, em 2016. A amostra foi definida pelo método de saturação de dados e constou de sete participantes. Para a coleta de dados utilizou-se a entrevista estruturada e como referencial metodológico a análise de conteúdo temática. A pesquisa iniciou-se após aprovação do projeto pelo Comitê de Ética em Pesquisa. Resultados: a partir dos discursos elencaram-se as categorias: expectativas profissionais antes da visitação aberta; enfrentando as dificuldades da visitação aberta; evidenciando os benefícios da visitação aberta; e desafios no seu aprimoramento. Conclusão: a percepção da equipe de enfermagem inicialmente foi negativa, porém após a implementação da visitação aberta na UTIN, evidenciaram-se os benefícios para os recém-nascidos, pais, acompanhantes e equipe.
\end{abstract}

Descritores: Enfermagem; unidade terapia intensiva neonatal; humanização da assistência; relações profissional-família.

\section{ABSTRACT}

Objective: to understand nursing personnel's experience of open visitation in a neonatal intensive care unit, before and after implementation. Method: this descriptive, qualitative study was conducted at a public maternity hospital in Bauru, São Paulo, Brazil, in 2016. The sample, defined by the data saturation method, consisted of seven participants. Data were collected by structured interviews, and thematic content analysis provided the methodological frame of reference. The study after approval by the research ethics committee. Results: the categories identified from the discourse were: professional expectations before open visitation; coping with the difficulties of open visitation; evidencing the benefits of open visitation; and challenges in improving it. Conclusion: the nursing team's initial perceptions were negative, but after implementation of open visitation, the benefits for newborns, parents, companions, and staff became evident.

Descriptors: Nursing; newborn intensive care units; humanization of care; professional-family relations.

\section{RESUMEN}

Objetivo: comprender la experiencia de profesionales de enfermería sobre la visitación abierta en una unidad de terapia intensiva neonatal (UTIN), antes y después de su implementación. Método: estudio descriptivo, cualitativo, desarrollado en una maternidad pública situada en Bauru, São Paulo, Brasil, en 2016. La muestra fue definida por el método de saturación de datos y consistió en siete participantes. Para la recolección de datos se utilizó la entrevista estructurada y, como referencial metodológico, el análisis de contenido temático. La investigación se inició tras la aprobación del Comité de Ética en Investigación. Resultados: Con base en los discursos, se enlistaron las categorías: expectativas profesionales antes de la visitación abierta; enfrentando las dificultades de la visitación abierta; evidenciando los beneficios de la visitación abierta; y desafíos en su perfeccionamiento. Conclusión: la percepción del equipo de enfermería inicialmente fue negativa pero, después de la implementación de la visitación abierta en la UTIN, se evidenciaron los beneficios a los recién nacidos, padres, acompañantes y equipo.

Descriptores: Enfermería; unidad terapia intensiva neonatal; humanización de la asistencia; relaciones profesionales-familia.

\section{INTRODUÇÃo}

A unidade de terapia intensiva (UTI) destina-se ao atendimento de pacientes que demandam cuidados complexos e especializados ${ }^{1}$. Seu ambiente é considerado estressante e gerador de uma atmosfera emocionalmente comprometida, tanto para os profissionais como para os pacientes e seus familiares. Na expectativa de torná-lo mais humanizado, diferentes políticas têm sido propostas por órgãos federais e de classes de profissionais².

Dentre os diversos contextos abordados nas políticas de humanização, destaca-se a valorização da pre-

'Enfermeiro. Hospital de Reabilitação de Anomalias Craniofaciais, Universidade de São Paulo. Brasil. E-mail: fabiolbanhara@hotmail.com

"Enfermeira. Mestre em Ciências, Programa de Pós-Graduação em Ciências da Reabilitação, Universidade de São Paulo. Brasil. E-mail: francelyfarinha@usp.br '"Enfermeira. Hospital de Reabilitação de Anomalias Craniofaciais, Universidade de São Paulo. Brasil. E-mail: tatianee henrique@hotmail.com

IVnfermeira. Doutora em Ciências. Professora Titular do Departamento de Enfermagem, Universidade Paulista, Bauru, São Paulo, Brasil.E-mail: anapaularazera@gmail.com vPediatra. Maternidade Santa Isabel, Fundação para o Desenvolvimento Médico e Hospitalar. Brasil. E-mail: narenales.msi@famesp.org.br

v'Enfermeiro. Doutor em Ciências. Hospital de Reabilitação de Anomalias Craniofaciais, Universidade de São Paulo. Brasil. E-mail: armandotrettene@usp.br 
sença de acompanhantes durante a internação, sendo considerado um processo fundamental para o alcance de uma prática assistencial realmente humanizada ${ }^{3,4}$.

Frente ao exposto, o objetivo do presente estudo foi compreender a experiência de profissionais de enfermagem sobre a visitação aberta em uma unidade de terapia intensiva neonatal (UTIN), antes a após sua implementação.

\section{REVISÃo DE LITERATURA}

Diferentes normativas estabelecem que os hospitais propiciem condições à permanência em tempo integral de um dos pais ou responsável nos casos de internação da criança ou adolescente ${ }^{3,4}$. Nesse contexto, o paciente deixou de ser o único foco da assistência em saúde, ressaltando-se a necessidade de inserção da família no processo de cuidar na enfermagem, principalmente pelo fato de estarem vinculados ao sucesso do processo de reabilitação e melhoria no prognóstico dos pacientes ${ }^{5,6}$.

Diferentes são as populações atendidas na UTI, incluindo-se o público pediátrico e neonatal, onde a necessidade de permanência dos pais é indispensável. Embora os benefícios referentes à permanência dos acompanhantes durante a internação sejam evidentes, sua implementação encontra-se aquém do ideal ${ }^{6,7}$. A instabilidade clínica, a complexidade dos cuidados e o risco de infecção são alguns dos motivos alegados pelos dirigentes e profissionais para se oporem a essa prática ${ }^{2,6}$. Contudo, as exigências por parte dos familiares, cada vez mais cientes de seus direitos, associadas à necessidade de cumprir o determinado pela legislação, têm universalizado essa prática, exigindo dos serviços a sua implementação.

Embora estudos sobre a percepção da equipe de enfermagem referentes à permanência dos pais na UTIN estejam disponíveis, investigações que comparem essa percepção antes e após a implementação da visitação aberta são incipientes.

\section{Metodologia}

Trata-se de um estudo descritivo, com enfoque qualitativo, desenvolvido em uma maternidade pública situada em Bauru, São Paulo, Brasil, em junho de 2016.

A população foi composta por profissionais de enfermagem, incluindo enfermeiros e técnicos de enfermagem. O critério de inclusão foi atuar na UTIN por período superior a um ano. A amostra intencional e por conveniência foi definida pelo método de saturação de dados ${ }^{8}$ e constou de sete participantes, sendo três enfermeiros e quatro técnicos de enfermagem.

A pesquisa iniciou-se após aprovação do projeto pelo Comitê de Ética em Pesquisa da Universidade Paulista, por meio do parecer 1.784 .675 e CAAE: 60181016.0.0000.5512. Os participantes formalizaram sua adesão mediante um Termo de Consentimento Livre e Esclarecido, em conformidade com os princípios éticos e legais vigentes. O estudo foi conduzido segun- do os critérios consolidados para relatos de pesquisa qualitativa - COREQ ${ }^{9}$.

Para a coleta de dados utilizou-se a entrevista estruturada. O elemento disparador foi: conte-me sobre sua percepção referente à visitação aberta na UTIN antes e após a sua implementação. Considerou-se como visitação aberta a possibilidade de permanência do acompanhante em tempo integral junto ao recém-nascido, durante sua permanência na UTIN.

As entrevistas ocorreram em ambiente privativo, fora do turno de trabalho e foram audiogravadas. 0 conteúdo das gravações foi transcrito visando facilitar o processo de análise dos dados emergentes, além de aprimorar e aprofundar a entrevista posterior ${ }^{8,10}$.

Para identificar a fala e garantir a manutenção do anonimato, utilizou-se a letra $\mathrm{P}$ de profissional para cada sujeito, bem como números arábicos sequenciais. O tempo médio de duração da entrevista foi de 30 minutos. Ainda, os participantes foram caracterizados segundo a idade, sexo, estado civil, categoria profissional, tempo de formação e tempo de atuação na UTIN.

Para a análise qualitativa, utilizou-se a metodologia da análise de conteúdo temática, em que o tratamento dos dados é realizado pela inferência e interpretação dos conteúdos, organizadas e similaridade, sendo sistematizado nas seguintes fases: pré-análise - ocorreu a leitura flutuante dos conteúdos das entrevistas por meio dos princípios da exaustividade, representatividade, homogeneidade e pertinência; exploração do material - houve a construção das operações de codificação, por meio da identificação de palavras-chave e temas correlatos, com posterior agregação das informações em categorias temáticas; interpretação - houve tratamento dos dados, inferência e intrepretação ${ }^{8,10}$.

\section{RESULTADOS E DiscusSÃo}

Participaram do presente estudo sete profissionais de enfermagem, sendo 3 (43\%) enfermeiros e 4 (57) técnicos de enfermagem. A média de idade foi 37 anos ( \pm 11$)$, todos do sexo feminino, sendo 5 (72\%) casadas e 6 (86\%) com filhos. O tempo médio de atuação na UTIN foi de 5 anos ( \pm 3 ) e o de formação profissional foi de 8 anos $( \pm 5)$.

A partir dos discursos elencaram-se quatro categorias: expectativas profissionais antes da visitação aberta, enfrentando as dificuldades da visitação aberta; evidenciando seus benefícios; e desafios no seu aprimoramento.

\section{Expectativas profissionais antes da visitação aberta}

Evidenciaram-se as expectativas referentes à maneira como os pais reagiriam frente aos procedimentos realizados com seus filhos, uma vez que não possuíam conhecimento sobre os mesmos. Ainda, observou-se a preocupação dos profissionais sobre as cobranças junto à equipe.

Tínhamos medo que os pais não compreendessem as intercorrências e atrapalhassem ou não permitissem re- 
alizar os procedimentos nos bebês [...] dos pais ficarem observando e cobrando. (P3)

Não estávamos acostumados a trabalhar com os pais assistindo nosso trabalho [...] não porque fizéssemos coisas erradas, mas eles não têm conhecimento dos procedimentos [...] é mais ansiedade, insegurança referente ao que eles acham do nosso trabalho. (P5)

A preocupação quanto à maneira como os pais reagiriam, frente aos procedimentos e condutas realizadas com seus filhos, refere-se à situação desfavorável deles, muitas vezes com estresse físico e psicológico diante da internação, uma vez que a UTIN representa um ambiente hostil, de gravidade clínica e risco de morte ${ }^{11,12}$.

A segurança dos profissionais em lidar com equipamentos e materiais, por vezes, é interpretada pelos pais, familiares e acompanhantes como de frieza, mecanicismo e desumanização. Nesse sentido, a hegemonia do conhecimento por parte dos profissionais tende a ser defendida ${ }^{12}$. Dessa forma, a assimilação de um novo modo de ser e estar na UTIN, baseada na corresponsabilização pelo cuidado e compartilhamento de saberes com os pais, encontra resistências. Contudo, entende-se que essas reações são formas de resistência e autoproteção ${ }^{13}$.

Os pais e acompanhantes possuem uma visão diferente quanto à realidade dos cuidados, particularmente frente aos procedimentos que podem causar dor ou desconforto aos recém-nascidos, sendo por eles considerados como maltrato e não como medida terapêutica necessária. Constata-se que a falta de conhecimento científico, bem como sobre a necessidade do procedimento, desencadeia nos pais insegurança, sofrimento e resistência. Assim, esclarecimentos sobre a necessidade das intervenções, emprego de técnicas para redução do desconforto do recém-nascido e preparo para os procedimentos tendem a diminuir o impacto negativo sobre os pais, gerando compreensão $0^{5,12,14}$.

A preocupação dos profissionais sobre possíveis dificuldades de acolhimento e acomodação dos familiares na UTIN também foi evidenciada, uma vez que o ambiente não foi planejado para acolhê-los continuadamente.

Ficávamos preocupados com os pais permanecerem $o$ tempo todo junto aos bebês, por que o espaço é limitado. Isso poderia tumultuar a UTIN. (P1)

Pensava em como seria a permanência dos pais em todo o tempo, pois a UTIN é apertada, os leitos são muito próximos, não temos como acomodá-los bem. (P6)

Algumas dificuldades são apontadas como restritivas à implementação da visitação aberta, incluindo espaço físico inadequado ${ }^{15,16}$. Apesar da relevância dada pela equipe a questões desse gênero, são apontados como de menor importância por familiares, que priorizam o acolhimento com informações precisas e claras sobre os pacientes ${ }^{17,18}$.

\section{Enfrentando as dificuldades da visitação aberta}

Os profissionais expressaram as dificuldades vivenciadas no processo de sua implementação, que incluíram as limitações de entendimento e falta de adesão por parte dos pais às normas e rotinas da UTIN, ocasionando conflitos com a equipe. Os pais geralmente apresentam-se ansiosos e preocupados, e a dificuldade de compreensão do todo implica a procura ou solicitação intensa da enfermagem gerando descontentamento nos profissionais.

A população que atendemos é muito carente e tem menos acesso a informações [...] são famílias sem orientação $e$ muitas vezes com problemas sociais. Isso dificulta bastante nosso trabalho, por que não entendem a necessidade dos procedimentos e as normas e rotinas da UTIN. (P3)

Existem pais que causam transtornos e não respeitam as normas [...]. (P4)

Outras dificuldades relatadas incluíram a preocupação da equipe em sentir-se observada durante a realização dos cuidados e a resistência ou descontentamento dos pais quanto à realização de procedimentos invasivos.

A maior dificuldade foi realizar os procedimentos com os pais observando [...] alguns mostram resistência em permitir a realização de exames e algumas vezes veem os procedimentos como maltrato ao bebê. (P1)

Sinto-me vigiada por eles [...] Perguntam muito! [...] Às vezes fazem a mesma pergunta para vários profissionais pra ver se estão falando a mesma coisa [...] Na equipe teve profissionais que ficaram irritados porque não estavam acostumados e se sentiam pressionados. (P3)

Profissionais de enfermagem tendem a retrair-se e considerar como invasiva a presença dos pais, alegando falta de espaço, interferência nas rotinas e descumprimento de normas, apontando a necessidade de treinamento da equipe para lidar com tais questões ${ }^{13,19}$.

A equipe de enfermagem frequentemente apresenta-se resistente quanto à oferta de informação aos familiares, o que pode acarretar sentimentos de insegurança e medo, gerando maior estresse aos pais e acompanhantes. Nesse contexto, ressalta-se a importância da orientação e do esclarecimento de dúvidas como intervenção eficaz junto aos familiares ${ }^{5,17,14,20}$.

Familiares e acompanhantes desejam receber informações do médico referente ao estado clínico de seus entes. Contudo, esperam receber informações do enfermeiro e sua equipe sobre os cuidados de higiene, alimentação, exames, entre outros ${ }^{21}$.

Outro aspecto a ser considerado refere-se à necessidade de se atentar para a comunicação não verbal, por oferecer indícios de necessidades não reveladas ${ }^{22}$. 0 fato de o hospital ser público, e, portanto, atender uma população desfavorável socialmente deve ser considerado, uma vez que a baixa escolaridade, por exemplo, pode influenciar o entendimento das comunicações.

Ressalta-se que na UTIN, campo do presente estudo, na admissão do recém-nascido é ofertado aos pais ou acompanhantes um folder contendo informações referentes à unidade, bem como suas normas e rotinas. Esse folder é lido pelo enfermeiro junto aos familiares, visando facilitar o entendimento, embora contenha linguagem 
acessível a leigos. No entanto, evidencia-se que alguns pais ou acompanhantes apresentam-se resistentes a seguir determinadas regras, incluindo o uso de adornos, celulares, curiosidade em visualizar os outros bebês, necessitando serem abordados constantemente pela equipe.

Disponibilizar material impresso, como folhetos explicativos, juntamente com informações claras, auxilia na compreensão das normas, rotinas e cuidados realizados na UTI, gerando maior aceitação e redução do estresse aos pais, acompanhantes e equipe ${ }^{23}$.

Quanto ao fato de os pais solicitarem informações a diversos profissionais como forma de validar ou testar a resposta obtida, infere-se que possa decorrer de falta de compreensão e não de desconfiança. Populações de baixa escolaridade apresentam dificuldades em compreender termos técnicos, recorrendo a outros membros da equipe para conseguir informações mais simplificadas ${ }^{24}$.

Estratégias são apresentadas visando facilitar a compreensão dos pais e melhorar a comunicação entre equipe e familiares, inclusive usar comparações de fácil compreensão, explicar termos técnicos, validar a informação com os pais, indagando-os sobre o que compreenderam, fornecer informações aos poucos, entre outras ${ }^{25,26}$.

\section{Evidenciando os benefícios da visitação aberta}

Em relação à equipe de enfermagem, os profissionais relataram mudanças nos comportamentos relativos à postura profissional.

A gente começa a se policiar [...] principalmente em relação às conversas e posturas, porque os pais prestam atenção no que falamos e como agimos. (P1)

O comportamento da equipe mudou pra melhor depois da visita aberta [...] Ficamos mais atentos para não passar uma imagem inadequada. (P2)

Outro benefício vinculado à visitação aberta referiu-se à maior confiança e segurança dos pais na equipe de enfermagem.

Quando eles estão dentro da unidade, veem o cuidado e deixam de pensar que o bebê possa estar sendo negligenciado ou mal cuidado [...] Valorizam mais nosso trabalho. (P7)

Quando o horário de visita era restrito, a cobrança e o medo dos acompanhantes eram maiores [...] eles não sabiam como estavam sendo cuidados seus filhos [...] agora ficaram mais confiantes e seguros em deixar seus filhos sob os cuidados da equipe. (P6)

A valorização da equipe decorre do processo de avaliação positiva pelos pais em relação aos cuidados, considerando-se o zelo, a interação com o recém-nascido durante os procedimentos técnicos e a oferta de informações adequadas aos familiares. Isso acontece necessariamente devido à observação da equipe pelos pais e, vice-versa, de modo a estabelecer um vínculo e conhecimento entre ambas as partes ${ }^{27,28}$.

Com a visitação aberta, os pais foram incentivados e inseridos nos cuidados de seus filhos, o que proporcio- nou a interação entre eles, fortalecendo a confiança dos mesmos na realização dos cuidados. Ressalta-se, ainda, o aprendizado dos pais ou acompanhantes quanto aos cuidados, que deverão ser continuados no domicílio ${ }^{29}$.

Constatou-se, também, que os pais se sentiram mais seguros e satisfeitos, uma vez que puderam acompanhar a realização dos procedimentos e comprovar a qualidade do cuidado.

Nós procuramos fazer com que eles participem dos procedimentos e buscamos esclarecer todas as suas dúvidas. Eles se apegam sempre a alguém [...] estabelecem uma relação de confiança. (P 1)

Para os pais, essa experiência foi positiva, pois não tem aquele desespero de ir embora e deixar o filho [...] os médicos e enfermeiros conversam em qualquer horário [...] eles têm acesso à informações, adequadamente. (P 2)

A partir do estabelecimento da relação de confiança entre os pais ou acompanhantes e a equipe de enfermagem, observam-se cumplicidade e entendimento, inclusive na realização de procedimentos invasivos junto aos recém-nascidos, uma vez que entendem a necessidade dos mesmos e que todos os esforços e intervenções visam à recuperação deles. Ressalta-se que o número reduzido de visitas, bem como o tempo limitado, relaciona-se com menor satisfação dos pais e familiares ${ }^{22}$.

A insensibilidade, a falta de oferta de informação e a indisponibilidade do profissional quando solicitado, assim como a verificação de um déficit na quantidade de funcionários, geram sentimentos de desamparo e tendem a favorecer a resistência do acompanhante à cooperação. Contudo, a observação constante e permanência na unidade tendem a tornar os pais mais compreensivos e colaborativos com a equipe ${ }^{11,27}$.

Quanto aos benefícios da visitação aberta para os neonatos, incluíram-se o fortalecimento do vínculo parental e o estímulo da sensibilidade dos neonatos ao estado emocional transmitido pelos pais.

Quando os pais estão presentes o bebê fica mais calmo [...] eles reconhecem a voz dos pais e percebemos uma melhor evolução. $O$ vínculo entre os pais e bebês aumenta [...] O essencial é o vínculo que é fortalecido. (P 2)

Os pais compreendem melhor a situação do filho $e$ ficam menos inseguros e ansiosos [...] se sentem mais preparados referente aos cuidados após a alta. (P 6)

O contato com o recém-nascido e o envolvimento dos pais nos cuidados têm como potencialidade fortalecer o vínculo e o apego que pode se encontrar abalado pela hospitalização ou com a figura do filho que foge aos padrões narcísicos de perfeição. O sentimento de culpa dos pais associado a estes casos pode gerar negligência, devendo ser trabalhado durante a hospitalização $0^{30,31}$.

Em contrapartida, a visitação aberta proporciona maior compreensão do estado clínico do recém-nascido, assim como dos procedimentos realizados, até mesmo nos casos cuja evolução é desfavorável. 
Quanto aos benefícios referentes aos recém-nascidos, além do fortalecimento do vínculo com seus pais, os profissionais relataram melhorias nas condições clínicas e aspectos emocionais dos pais e bebês. É de extrema importância incluir as famílias nas decisões terapêuticas e no cuidado de seus filhos de modo a promover uma relação terapêutica positiva ${ }^{29,32}$. Horários expandidos ou flexíveis de visitação são apontados como mais importantes que sua duração, por proporcionar adaptações às necessidades de cada caso em particular ${ }^{6}$.

O método canguru, com a participação dos pais como acompanhantes com livre acesso à UTIN, é um dos pilares; pesquisa realizada com o objetivo de avaliar seus resultados no Brasil, constatou benefícios referentes à promoção e manutenção do aleitamento materno ${ }^{33}$. Outras vantagens dessa metodologia incluem a redução do tempo de internação e da mortalidade neonatal ${ }^{24}$. Esses resultados evidenciam alguns dos benefícios da visitação aberta.

\section{Desafios no aprimoramento da visitação aberta}

Os profissionais discorreram sobre os desafios referentes ao espaço físico - proporcionar conforto aos pais e familiares e aperfeiçoar o processo de acolhimento.

Deveriam ser oferecidos cadeiras confortáveis e banheiros próximos para maior comodidade [...] melhorar a oferta de informação por parte da equipe e dar mais atenção. (P 3)

É necessário um pouco mais de espaço, uma recepção ou sala de espera [...] alguma orientação antes de entrarem na UTIN, principalmente na primeira vez. (P 2)

Deveria ter um grupo de reunião com os pais com uma equipe multidisciplinar [...] deveria trabalhar mais os pais, dar mais informação, apoio psicológico [...]. (P 5)

Como impedimento à visitação aberta, entre outros, inclui-se a ausência de espaço apropriado para os acompanhantes junto aos pacientes. Investigação apontou que $51 \%$ das UTI brasileiras não oferecem qualquer comodidade aos familiares ${ }^{6}$. Entre as sugestões propostas para melhorar o processo de acolhimento na UTIN, inclui-se $o$ atendimento por um grupo multidisciplinar de apoio aos pais e familiares ${ }^{6,17}$.

Embora a legislação garanta condições apropriadas à permanência dos pais e acompanhantes por período integral, na prática percebe-se que esta determinação está aquém de ser cumprida, representando um grande desafio aos gestores e à equipe de saúde $\mathrm{de}^{3,4,24}$.

\section{CONCLUSÃo}

As expectativas profissionais da equipe de enfermagem referente à visitação aberta em UTIN inicialmente foram negativas, ocasionando insegurança e resistência. Posteriormente, com a sua implementação, as dificuldades foram enfrentadas e minimizadas, e, por fim, evidenciaram-se benefícios para os recém-nascidos, pais, acompanhantes e equipe. Contudo, evidenciou-se a necessidade de se aprimorar aspectos referentes ao acolhimento e conforto aos pais e acompanhantes. Em suma, a implementação da visitação aberta pode ser referida com um processo onde a equipe de enfermagem é protagonista.

Espera-se que os achados desta investigação contribuam para desmistificar aspectos da implementação da visitação aberta, em UTIN, e embasem estratégias para a sua falsificação e seu aperfeiçoamento.

Como limitação, aponta-se a característica monocêntrica deste estudo que não permite a generalização dos resultados. Assim, considerando-se a relevância dessa temática, a realização de outros estudos em diferentes contextos é encorajada.

\section{REFERÊNCIAS}

1. Siqueira HCH, Medeiros AC, Zamberlan C. Configuração da gestão do cuidado de enfermagem na UTI. In: Sousa FGM, Backes DS, organizadores. Cuidado em enfermagem e saúde: diversidades e complexidades. Florianópolis (SC): Papa Livro; 2015. p. 307-35.

2. Roseiro CP, Paula KMP. Concepções de humanização de profissionais em Unidades de Terapia Intensiva Neonatal. Estud. Psicol. (Campinas, Online). [Scielo-Scientific Library Online]. 2015 [citado 2017 dez 20];32(1):109-19. Disponível em: http://www.scielo.br/ pdf/estpsi/v32n1/0103-166X-estpsi-32-01-00109.pdf

3. Ministério da Saúde (Br). Estatuto da criança e do adolescente. Brasília (DF). [Internet] 2017. [citado 2017 dez 20]; Disponível em: http://www.chegadetrabalhoinfantil.org.br/wp-content/ uploads/2017/06/LivroECA_2017_v05_INTERNET.pdf

4. Ministério da Saúde (Br). Portaria no 1.153 de 22 de maio de 2014. Redefine os critérios de habilitação da Iniciativa Hospital Amigo da Criança (IHAC). [Internet] 2014 [citado 2017 dez 20]; Disponível em: http://bvsms.saude.gov.br/bvs/saudelegis/gm/2014/ prt1153_22_05_2014.html

5. Lima AS, Silva VKBA, Collet N, Reichert APS, Oliveira BRG. Relações estabelecidas pelas enfermeiras com a família durante a hospitalização infantil. Texto \& contexto enferm. (Online). [Scielo-Scientific Library Online]. 2010 [citado 2017 dez 20];19(4):700-8. Disponível em: www.scielo.br/pdf/tce/v19n4/13.pdf

6. Ramos FJS, Fumis RR, Azevedo LC, Schettino G. Intensive care unit visitation policies in Brazil: a multicenter survey. Rev. bras. ter. Intensiva. [Scielo-Scientific Library Online]. 2014 [accessed 2017 Dec 20]; 26(4):339-46. Available from: www.scielo.br/pdf/ rbti/.../en_0103-507X-rbti-26-04-0328.pdf

7. Garrouste-Orgeas M, Philippart F, Timsit JF, Diaw F, Willems V, Tabah A, et al. Perceptions of a 24-hour visiting policy in the intensive care unit. Crit. care med. [PubMed-NCBI]. 2008 [accessed 2017 Dec 20]; 36(1):30-5. Available from: https://www.ncbi. nlm.nih.gov/pubmed/18090167

8. Minayo MCS. O desafio do conhecimento: pesquisa qualitativa em saúde. 14ạ ed. São Paulo: Hucitec; 2014.

9. Tong A, Sainsbury P, Craig J. Consolidated criteria for reporting qualitative research (COREQ): a 32-item checklist for interviews and focus groups. Int. j. qual. health care. [PubMed-NCBI]. 2007 [accessed 2017 Dec 20];19(6):349-57. Available from: https:// academic.oup.com/intqhc/article/19/6/349/1791966

10. Bardin L. Análise de conteúdo. 5ạ ed. Lisboa (Pt): Edições 70; 2013.

11. Luz CF, Melnik MG, Bernardino E, Oliveira ES. Compreendendo as restrições dos técnicos de enfermagem sobre a permanência de acompanhantes em Unidade de Terapia Intensiva aberta. Texto \& contexto enferm. (Online). [Scielo- Scientific Library Online]. 2009 [acessado 20 dez 2017]; 18(2):306-12. Disponível em: www.scielo. $\mathrm{br} / \mathrm{pdf} / \mathrm{tce} / \mathrm{v} 18 \mathrm{n} 2 / 14 . \mathrm{pdf}$

12. Custodio N, Marski BSL, Abreu FCP, Mello DF, Wernet M. 
Interações entre profissionais de saúde e mães de prematuros: influência no cuidado materno. Rev. enferm. UERJ. [Internet]. 2016 [acessado 20 ago 2017]; 24(1):e11659. Disponível em: http:// www.facenf.uerj.br/v24n1/v24n1a05.pdf

13. Costa R, Padilha MI. Health care team perceptions of the family in the neonatal ICU: resistence to new knowledge. Rev. enferm. UERJ. [Internet]. 2011 [accessed 2017 Dec 20]; 19(2):231-5. Available from: www.facenf.uerj.br/v19n2/v19n2a10.pdf

14. Nascimentol ERP, Gulini JEHMB, Minuzzi AP, Rasia MA, Danczuk RFT, Souza BC. As relações da enfermagem na unidade de terapia intensiva no olhar de Paterson e Zderad. Rev. enferm. UERJ. [Internet]. 2016 [acessado em 20 ago 2017]; 24(2):e5817. Disponível em: http://www.facenf.uerj.br/v24n2/v24n2a16.pdf 15. Slota M, Shearn D, Potersnak K, Haas L. Perspectives on family-centered, flexible visitation in the intensive care unit setting. Crit. care med. [PubMed-NCBI]. 2003 [accessed 2017 Dec 20]; 31(5Suppl):S362-6. Available from: https://www.ncbi.nlm.nih. gov/pubmed/12771584

16. Berti D, Ferdinande P, Moons P. Beliefs and attitudes of intensive care nurses toward visits and open visiting policy. Intensive care Med. [PubMed-NCBI]. 2007 [accessed 2017 Dec 20];33(6):1060-5. Available from: https://www.ncbi.nlm.nih.gov/ pubmed/17384930

17. Barth AA, Weigel BD, Dummer CD, Machado KC, Tisott TM. Stressors in the relatives of patients admitted to an intensive care unit. Rev. bras. ter. Intensiva. [Scielo- Scientific Library Online]. 2016 [accessed 2017 Dec 20]; 28(3):323-9. Available from: www. scielo.br/pdf/rbti/v28n3/0103-507X-rbti-28-03-0323.pdf

18. Reis LS, Silva EF, Waterkemper R, Lorenzini E, Cecchetto FE. Humanization of healthcare: perception of a nursing team in a neonatal and pediatric intensive care unit. Rev. gaúch. enferm. [Scielo-Scientific Library Online]. 2013 [accessed 2017 Dec 20]; 34(2):118-24. Available from: https://www.scielo.br/scielo. php?script=sci_arttext\&pid=S1983-14472013000200015

19. Ramos FJS, Fumis RR, Azevedo LC, Schettino G. Perceptions of an open visitation policy by intensive care unit workers. Annals. of Intensive Care [PubMed-NCBI]. 2013 [accessed 2017 Dec 20]; 3(1):34. Available from: https://www.ncbi.nlm.nih.gov/pmc/ articles/PMC3854481

20. Passos SSS, Silva JO, Santana VS, Nascimento VM, Álvaro PereiraV, Santos LM. O acolhimento no cuidado à família numa unidade de terapia intensiva. Rev. enferm. UERJ. [Internet]. 2015 [acessado em 20 ago 2017]; 23(3):368-74. Disponível em: http:// www.e-publicacoes.uerj.br/index.php/enfermagemuerj/article/ view/6259

21. Trettene AS, Fontes CMB, Razera APR, Gomide MR. Impact of promoting self-care in nursing workload. Rev. Esc. Enferm. USP. [Scielo-Scientific Library Online]. 2016 [accessed 2017 Aug 15]; 50(4):635-41. Available from: http://www.scielo.br/pdf/reeusp/ v50n4/0080-6234-reeusp-50-04-0635.pdf

22. Puggina AC, lenne A, Carbonari KFBSF, Parejo LS, Sapatini TF, Silva MJP. Perception of communication, satisfaction and importance of family needs in the Intensive Care Unit. Esc Anna Nery Rev. Enferm. [Scielo-Scientific Library Online]. 2014 [accessed 2017 Dec 20];18(2):277-83. Available from: www.scielo.br/pdf/ ean/v18n2/en_1414-8145-ean-18-02-0277.pdf

23. Pelazza BB, Simoni RC, Freitas EG, Silva BR, Silva MJ. Nursing visit and doubts expressed by families in the Intensive Care Unit.
Acta Paul. Enferm. (Online). [Scielo-Scientific Library Online]. 2015 [accessed 2017 Dec 20];28(1):60-5. Available from: www.scielo. br/pdf/ape/.../en_1982-0194-ape-028-001-0060.pdf

24. Soares LO, Santos RF, Gasparino RC. Necessidades de familiares de pacientes internados em unidade intensiva neonatal. Texto \& contexto enferm. (Online). [Scielo-Scientific Library Online]. 2010 [acessado em 20 dez 2017]; 19(4):644-50. Disponível em: www. scielo.br/pdf/tce/v19n4/06.pdf

25. Redshaw ME, Harvey ME. Explanations and information-giving: clinician strategies used in talking to parents of preterm infants. BMC pediatrics. (Online). [Internet]. 2016 [accessed 2017 Aug 15]; 16(1):25. Available from: https://bmcpediatr.biomedcentral.com/ track/pdf/10.1186/s12887-016-0561-6

26. Boss RD, Donohue PK, Larson SM, Arnold RM, Roter DL. Family Conferences in the Neonatal Intensive Care Unit: Observation of Communication Dynamics and Contributions. Pediatr Crit. care med. [PubMed-NCBI]. 2016 [accessed 2017 Dec 20]; 17(3):223-30. Available from: https://www.ncbi.nlm.nih.gov/ pubmed/26684988

27. Gomes GC, Xavier DM, Pintanel AC, Farias DHR, Lunardi VL, Aquino DR. Meanings attributed by family members in pediatrics regarding their interactions with nursing professional. Rev. Esc. Enferm. USP. [PubMed-NCBI]. 2015 [accessed 2017 Dec 20]; 49(6):953-9. Available from: https://www.ncbi.nlm.nih.gov/ pubmed/27419679

28. Machado NA, Sousa MLXF, Silva MEA; Coutinho SED, Reichert APS, Collet N. Dificuldades para a efetivação do acolhimento hospitalar durante a internação de crianças com doença crônica. Rev. enferm. UERJ. [Internet]. 2015 [acessado 20 ago 2017]; 23(4):556-61. Disponível em: http://www.e-publicacoes.uerj.br/ index.php/enfermagemuerj/article/view/5960/14250

29. Mondini CCSD, Fontes CMB, Trettene AS, Cianciarullo TI, Lazarini IM. Applicability of Orem: training of caregiver of infant with Robin Sequence. Rev. bras. enferm. (Online) [Scielo-Scientific Library Online]. 2018 [accessed 2017 Aug 15]; 71(Suppl.3):1469-73. Available from: http://www.scielo.br/pdf/reben/ v71s3/0034-7167-reben-71-s3-1469.pdf

30. Ministério da Saúde (Br). Secretaria de Atenção à Saúde. Departamento de Ações Programáticas Estratégicas. Atenção humanizada ao recém-nascido de baixo peso: Método Canguru. Manual Técnico. 2a ed. Brasília (DF): Ministério da Saúde; 2011. 31. Sikora K, Janusz B. Maternal bond with cardiosurgically treated infant. Qualitative analysis of mothers' narratives. See comment in PubMed Commons belowDev. Period. Med. [PubMed-NCBI]. 2014 [accessed 2017 Dec 20]; 18(4):439-46. Available from: https://www.researchgate.net/publication/271134806_Maternal_bond_with_cardiosurgically_treated_infant_Qualitative_analysis_of_Mothers'_narratives

32. Craig JW, Glick C, Phillips R, Hall SL, Smith J, Browne J. Recommendations for involving the family in developmental care of the NICU baby. J. perinatol. [PubMed-NCBI]. 2015 [accessed 2017 Dec 20]; 35(Suppl1):S5-8. Available from: https://www.ncbi.nlm.nih. gov/pmc/articles/PMC4660048

33. Lamy Filho F, da Silva AAM, Lamy ZC, Gomes MASM, Moreira MEL. Evaluation of kangaroo mother method in Brazil. J. Pediatr. (Rio J.) [PubMed-NCBI]. 2008 [accessed 2017 Dec 20]; 84(5):428-35. Available from: https://www.ncbi.nlm.nih.gov/ pubmed/18923784 